\title{
iTETRIS: Adaptation of ITS Technologies for Large Scale Integrated Simulation
}

\author{
Vineet Kumar ${ }^{1}$, Lan Lin $^{1}$, Daniel Krajzewicz ${ }^{2}$, Fatma Hrizi ${ }^{3}$, Oscar Martinez ${ }^{4}$, Javier Gozalvez ${ }^{4}$, Ramon Bauza ${ }^{4}$ \\ ${ }^{1}$ Hitachi Europe SAS, Sophia Antipolis, France \{Vineet.Kumar, Lan.Lin\}@hitachi-eu.com; \\ ${ }^{2}$ Deutsches Zentrum für Luft- und Raumfahrt e.V. (DLR), Berlin, Germany \{Daniel.Krajzewicz\}@dlr.de; \\ ${ }^{3}$ EURECOM, Sophia Antipolis, France \{Fatma.Hrizi\}@eurecom.fr \\ ${ }^{4}$ University Miguel Hernandez (UMH), Elche, Spain \{oscar.martinez, j.gozalvez, rbauza $@$ umh.es
}

\begin{abstract}
European Union (EU) Framework Program 7 (FP7) funded project, iTETRIS [1] (An Integrated Wireless and Traffic Platform for Real-Time Road Traffic Management Solutions) targets to extend state of art in simulation of wireless vehicular cooperative systems for evaluation of road traffic management services and applications. In particular, iTETRIS addresses four important and distinct challenges: (a) road traffic and wireless integrated open-source simulation platform, (b) large scale trials, (c) realistic Vehicle to Vehicle (V2V) and Vehicle to Infrastructure (V2I) communication simulation and (d) dynamic, distributed and self-autonomous Intelligent Transport Systems (ITS) [2] applications based on cooperative systems. iTETRIS will provide a standard compliant, open-source integrated communication and traffic platform suited for large scale scenario simulation.
\end{abstract}

This Paper addresses extension of traffic simulator being used in iTETRIS, scalability studies of networking simulator, which have been achieved as project results. Further the paper presents the adaptation of the Intelligent Communications for Transport Systems (ICTS) [2] reference architecture functionalities being developed by the European Telecommunication Standard Institute (ETSI) at its Technical Committee (TC) on ITS.

Keywords- Intelligent Transport Systems, Large Scale Integrated Simulation, Real-Time Traffic Management, V2X Cooperative Systems, network simulation.

\section{INTRODUCTION - ITETRIS SIMULATION ARCHITECTURE}

Although cooperative vehicular systems have been identified as a potential solution to improve traffic management and routing policies, the operational characteristics of such environment need to be designed and optimized. iTETRIS is devoted to address these needs and targets to provide a large scale $(\sim 20,000$ vehicles $)$ simulation platform to evaluate ITS strategies based on V2X cooperative systems in a close-to-real environment.

iTETRIS proposes a flexible 3-block Simulation architecture, a real-time closed-loop coupling between the traffic simulator, Simulation of Urban Mobility (SUMO) [3][4] and the Network Simulator 3 (ns-3) [5][6]. The integrated

\footnotetext{
${ }^{1}$ This paper also includes contributions from all the iTETRIS Consortium Partners: Thales Communications S.A., France (Project Coordinator); Hitachi Europe SAS, France; CBT Comunicación \& Multimedia, Spain; Comune di Bologna, Italy; German Aerospace Center (DLR), Germany; Innovalia Association, Spain; Peek Traffic B.V., The Netherlands; EURECOM, France; Universidad Miguel Hernandez (UMH), Spain. Project duration - 30 months, started on July, 2008.
}

system also proposes to provide a central control block to realize the coupling called 'iTETRIS Control System' (iCS). iCS would provide a platform for Application developers to develop and integrate their novel applications as well as an user interface to facilitate the usability of the platform by various section of users without any knowledge of the hidden kernel (ns-3 and SUMO). Flexibility and extendibility are main motivations of such architecture definition.

An overview of the iTETRIS 3-Blocks architecture and a representation of its intended users and developers of the platform are shown by Fig. 1 .

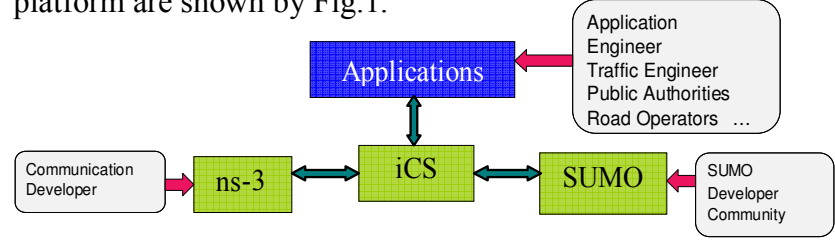

Fig 1.Targeted developers (ns-3, SUMO, Application Developers) / Users of the platform (traffic engineers, road authorities, general platform users)

The designed architecture can be seen as a logical extension of an already existing coupling between SUMO and ns-2, named TraNS [7][8]. TraNS was developed to couple SUMO and ns-2, the predecessor of ns-3. While still being popular, TraNS has the problem of being no longer actively supported since 2008 what disallows its usage with current versions of the coupled simulator SUMO. Furthermore, it lacks support for ETSI-compliant facilities and for including new applications, extensions to the used simulators as well as to TraNS itself are necessary. The iCS approach externalizes all application logics to simulate and support only application programmer interfaces (APIs) for accessing the used simulators. This allows implementing the application to simulate in any programming language as long as it supports socket communication.

In order to validate the platform and demonstrates its capability for traffic management strategies, traffic management center of Bologna (Italy) is contributing to the selection of applications for iTETRIS projects. Based on the local traffic characteristics and requirements of traffic management, 10 strategies are selected by iTETRIS to realize the simulation and application evaluation. These 10 strategies/applications are applied to several representative scenarios, such as big event management, highway congestion management, city ring road traffic management and complicated intersection management. 


\section{SimULATING ROAD TRAFFIC USING SUMO}

SUMO ("Simulation of Urban MObility") is an open source, microscopic road traffic simulation developed at the German Aerospace Center since 2001. SUMO has been successfully used within several projects for traffic management. Probably because of being available as open source, SUMO is often the first choice of institutions which are not originally involved in traffic science, but who need some basic characteristics of a real traffic's behavior. Due to this, SUMO is often used by wireless communication researchers, while traffic scientists still tend to use well-established commercial simulators such as Vissim, AIMSUN, or Paramics.

For evaluating the effects of traffic management methods or strategies, a microscopic road network simulation is needed, because both macro- and mesoscopic simulations do not allow retrieving the simulated vehicles' positions. These positions are essential information for computing the connectivity between communication participants in Vehicular Ad hoc Networks (VANET). The vehicle's positions, velocities, accelerations

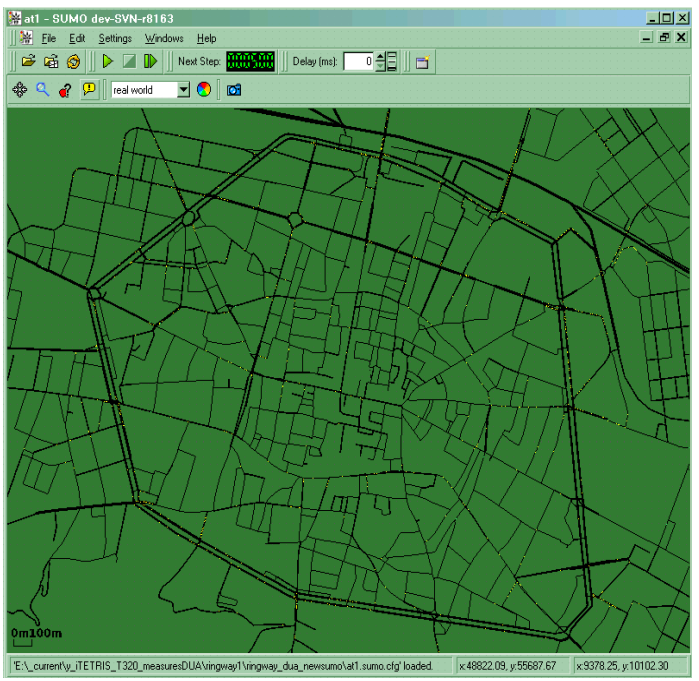

Fig 6. Simulation of Bologna's inner-city ring

and/or trajectories are also needed for simulating some traffic management strategies, such as green light speed advice. In this strategy, Road side station equipped at traffic lights broadcast the traffic light phase/timing information to the oncoming vehicles to calculate the optimized speed.

Because of being designed to simulate the behavior of a synthetic population in large scale, urban road networks, SUMO is able to simulate around 200.000 vehicle movements in real time on a modern desktop PC and no restrictions are put on the network size or on the number of simulated vehicles despite the used computer's memory. These qualities make SUMO a proper tool for evaluation of large-scale traffic management strategies.

Within the iTETRIS project, SUMO was extended to meet the needs posed by the project. Because iTETRIS puts a strong emphasis on developing strategies which bring an ecological benefit, i.e. reduce pollutant emissions or fuel consumption, SUMO was extended by the possibility to compute and write the amount of $\mathrm{CO}, \mathrm{CO}_{2}, \mathrm{HC}, \mathrm{PMx}$, and $\mathrm{NOx}$ emissions emitted by the simulated vehicles as well as their fuel consumption. The used model was derived using the "HBEFA" database [9] which includes data about vehicle emissions for 130 vehicle classes, divided by the size (passenger, light duty and heavy duty vehicles), by the fuel type they use, by engine displacement, and Euro-norm, for different modes of travel. The values from this database were fitted using a common emission function given below, first. The obtained curves' parameter were then clustered in order to obtain a lower number of (abstract) vehicle classes for simplifying the set-up of a vehicle population for a given scenario.

$e(v, a)=\frac{1}{h \gamma}\left(P_{0}+m a v+m g \mu_{0} v+m g \beta v+m g \mu_{1} v^{2}+\frac{1}{2} c_{w} \rho A v^{3}\right)$ with,

$\begin{array}{ll}c_{w} & \text { aerodynamic drag resistance } \\ m & \text { vehicle mass } \\ \mu_{0}, \mu_{1} & \text { (static and dynamic) friction coefficient } \\ P_{0} & \text { idle power consumption } \\ \gamma & \text { efficiency of the engine } \\ A & \text { front area of the vehicle } \\ h & \text { energy content of the fuel } \\ \rho & \text { density of the air } \\ g & \text { gravitational constant } \\ \beta & \text { slope of the road }\end{array}$

Furthermore, SUMO was extended by the Harmonoise noise emission model [10]. A visualization example is given in Fig.7.

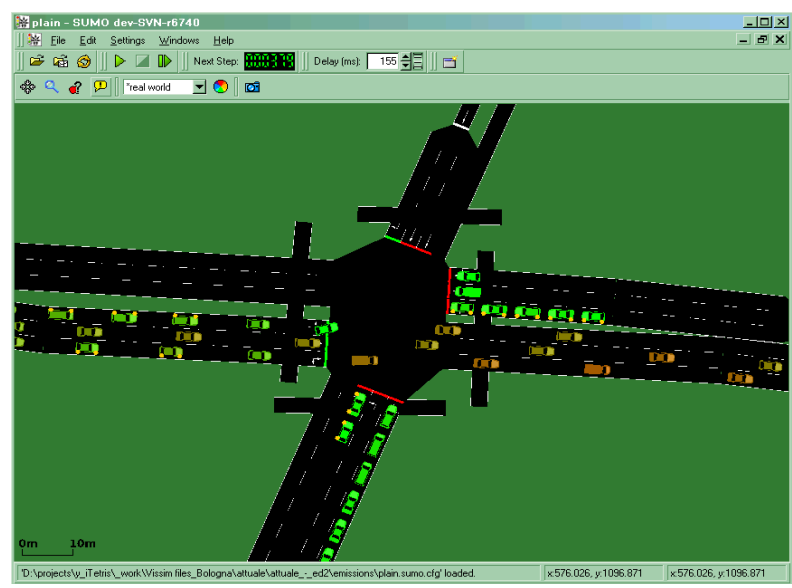

Fig 7. Visualisation of $\mathrm{CO}_{2}$ emissions

In subsequent iTETRIS tasks, further extensions were implemented, divided into the topics "intelligent rerouting", "intelligent traffic lights", and "advanced driver assistance systems". Most of this work concerned extending SUMO's accessibility by an external application by implementing 
interfaces for reading values of simulated structures, such as induction loops, traffic lights, and vehicles as well as interfaces for changing these structures' behavior. As already stated, these interfaces allow implementing the logic of a method to evaluate in any programming language and use it to control the simulation's behavior. The interfaces were implemented by extending SUMO's already available communication sub-system named "TraCI" [11][12] which was originally implemented by staff member of the technical university of Lübeck.

A further work package is concerned with allowing SUMO to simulate with steps $<1 \mathrm{~s}$. Two reasons make this necessary. At first, wireless communication simulation is normally performed using very fine grained time steps and lowering the simulation time steps is here necessary for a better synchronization of both simulators. Additionally, modern traffic flow models are often using steps of $0.1 \mathrm{~s}$. Allowing SUMO to use lower time steps is assumed to improve the simulation quality by enabling the usage of such models and also allows their evaluation in the context of road traffic simulation research.

\section{ITETRIS LARGE SCALE REQUIREMENTS: STUDY ON LARGE SCALE CAPABILITIES OF NS-3}

Considering the large scale requirements of iTETRIS, scalability has been the major concern in the choice of network simulator. For selecting the suitable network simulator, a comparative study was done on three widespread network simulators: ns-3, ns-2 [13] and OMNeT++ [14] because they suit the basic parameters required for the development of the iTETRIS project. The three network simulators were analyzed and compared with regard to several critical aspects. Although ns- 2 and OMNET++ have been widely used by the scientific community for simulation research over the past years, the relatively new ns-3 is intended to be the successor of ns-2. In fact, the number of ns-3 users is growing every month as the official website ns-3 downloads shows. Besides, ns-3 has demonstrated in our study high scalability capabilities and presents a more modular architecture, multi-technology support and superior future prospects in comparison with the others simulators. Regarding the simulation capabilities for large-scale scenarios, whereas ns-3 is capable of running simulation with more than 20000 wireless mobile nodes, ns-2 (version 2.33) was not able to simulate more than 8000 . Furthermore, ns- 2 requires a greater RAM memory consumption compared to ns-3 when considering high number of nodes. For these reasons, ns-3 has been selected as the iTETRIS wireless network simulation platform. Within iTETRIS project phase, the following access technologies will be developed and evaluated: ITS G5A, UMTS, WiMAX and DVB-H.

In spite of the ns-3 scalability capabilities, additional enhancements and performance improvements are needed to meet the demanding requirements of iTETRIS. It is convenient to highlight that iTETRIS is aimed to simulate city-sized scenarios and heterogeneous environments where multiple vehicles transmit/receive messages over more than one technology at the same time. Consequently, execution times are expected to be considerably high. Although on-going effort is being made by ns-3 developers to provide the simulator with support for parallelized simulations (simulation over several computers), it is not clear whether this technique will speed up wireless networks simulations. In this context, iTETRIS has developed and evaluated a set of optimization solutions to improve simulation performance, mostly aimed at simplifying physical layer complexity. For instance, the management of the interfering transmissions has been improved achieving up to $30 \%$ of performance improvement. Besides, the procedure for calculating the number of interfering nodes has also been optimized in order to prevent vehicles to process packets transmitted by distant nodes. An interference range has been included. The benefits of this technique can be observed in Fig. 4. The results are for $1 \mathrm{~h}$ emulation time, 20000 and 10000 nodes, different interference ranges $(700 \mathrm{~m}, 400 \mathrm{~m}$ and $100 \mathrm{~m})$ and high traffic density.

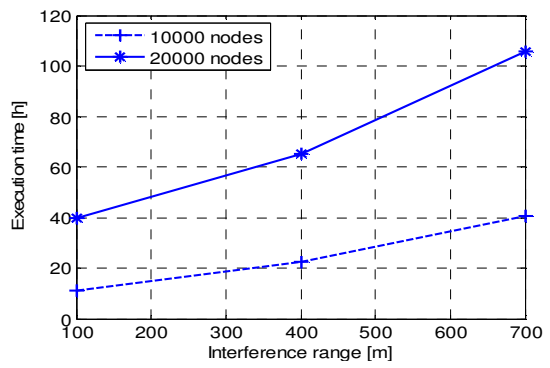

Fig. 4: Performance improvement by limiting the interference range.

Another factor directly impacting on the simulation performance estimation is related to the packet rate employed for the transmission of periodic messages, e.g CAM. When reducing the CAM rate from 10 to 2 packets per second, $80 \%$ execution time improvement is achieved. Further reductions in execution times can be obtained with, for instance, more powerful computational resources, code optimization, and static library compilation and region restrict communication simulation.

\section{ITETRIS COMMUNICATION ARCHITECTURE:}

\section{ADAPTATION OF ICTS REFERENCE ARCHITECTURE}

iTETRIS adheres to the objective to maintain constant effort to be in compliance with reference communication architectures being developed at ETSI TC ITS. Based on the constraints involved with large scale integrated simulations,

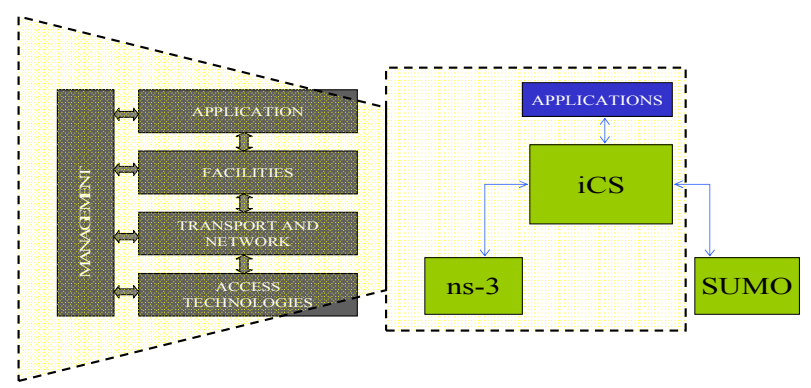

Fig 2. Adaptation of ICTS Communication Architecture by iTETRIS 3-blocks Integrated Simulation Architecture.

iTETRIS accordingly adapts to the reference architectures to suit its objectives. Fig. 2 demonstrates the adaptation of the ICTS communication architecture by the iTETRIS 3-Blocks architecture's communication and networking components. 
The ITS 'Applications' and 'Facilities' are included in the iTETRIS Application and iCS respectively, whereas the ITS 'Transport and Network', 'Management' and the 'Access technologies' are present in the ns-3. For computational optimization (adhering to the large scale simulation requirements of the iTETRIS platform), the implementation approach of the ITS Facilities' message management will be shared between iCS and ns-3.

\section{A. Adaptation of ITS facilities' for large scale integrated simulation: Communication and Application Related Facilities in iTETRIS.}

Facility layer is a supporting layer defined by ETSI for cooperative systems. It covers 5-7 OSI layers [15] and provides generic functions to cooperate with Transport \& Networking layers and to support ITS applications. ETSI defines three basic components in this layer:

"Application support" facilities and "information support" facilities are responsible for data management and application support functions, while "communication support" facilities aim to achieve applications' requirements in terms of communication modes.

iTETRIS' 3-Blocks architecture design complements the efforts to reduce computational time and resources by intelligent placement of modules to reduce inter-block message exchanges. Given this consideration, facilities layer as defined within ETSI is shared between iCS and ns-3. ns-3 will contain communication-related facilities. On the other hand, iCS implements Application-related facilities that are all the facilities which are closer to the application. Basically, it contains both "application support" and "information support" facilities. This aims to reduce as much as possible messages exchanges between these two blocks. The facility components are modified accordingly to function efficiently across the communication module spread across iCS and ns-3.

Appropriate adaptation of the ITS facilities for an efficient large scale simulation (iTETRIS) can be demonstrated by referring to the methodology adapted for implementation of the communication-related Message Management Facility.

iTETRIS' Message Management facility is responsible for handling exchange of V2X messages for cooperative communication. Examples of some of the safety and traffic efficiency cooperative messages proposed to be included within iTETRIS are Cooperative Awareness Message (CAM) and Decentralized Environmental Notification Message (DENM) proposed by ETSI.

The Message Management block is divided between the iCS and ns-3. The idea is to maintain a record of transmitted message payload data in iCS but not sending the actual payload to the message management component in ns-3 and subsequently to the transport layer (in ns-3) and further down the stack to be transmitted. Here. iTETRIS exploits the capability of ns-3 to create packets with 'dummy' payloads (without actual payload data) of specified size. In addition, iCS's Message Management also maintains a track of sent payloads and identifiers to locate on reception by the destination node. Subsequently on reception, the actual payload is obtained by the destination node by doing a simple look-up and delivered to the application. This method prevents excessive and large (in terms of size of data exchange) interactions between ns-3 and iCS, thus minimizing computation resources. This approach would limit the interaction between ns-3 and iCS, to only when it is necessary i.e when a facility message content (eg. DENM) has to be modified. Also the method abides to the 'classical' notion of a layered network architecture, according to which a forwarding node is not suppose to be aware of the payload content, unless it the intended destination, in which case it passes up to the application (or the 'Facilities' located at iCS in iTETRIS)

\section{B. VANET Protocols for iTETRIS: Adaptation for a computationally efficient large scale simulation}

iTETRIS intends to propose geo-networking non-IP stack (iTETRIS C2C stack) along with the existing IPv4 and IPv6 stacks. iTETRIS C2C stack would embed within a combination of basic and advanced (with topology awareness, advanced routing capabilities etc.) geo-networking protocols. iTETRIS has built liaisons with other on-going EU projects and standardization bodies. For development of the iTETRIS' basic geo-networking, it follows the specifications proposed by the on-going EU project, GeoNet [16]. The basic geo-routing protocols' implementations, intended to be included within iTETRIS platform are geo-unicast, geo-anycast, geo-broadcast and topo-broadcast. In addition, iTETRIS also proposes innovative mechanisms to enhance the routing capability of classical geo-routing protocols.

Advanced geo-broadcast routing schemes focus basically on ensuring reliability in multi-hop message dissemination. For instance, BZB [17] uses a distance-based strategy to select the forwarder node in order to cover the entire area. Other geo-routing protocols, also planned to be implemented are eg. GPSR, MOPR etc. Another mechanism that will be adopted is 'Intelligent Geo-routing'. This concept enables nodes to self-estimate the road topology and the neighbors' heading direction making use of network beaconing in the absence of digital maps. The neighbor data collected by this process is then statistically analyzed by each vehicle and subsequently, information like the number of neighbors, direction of traffic can be estimated by each node, this leads to intersection identification which helps efficient forwarding of packets. In addition, by Intelligent Geo-routing, vehicles can also be able to identify curves in the road topology. Furthermore, all above mentioned protocols will be studied and extended (if possible) to have a better V2V/V2I integration.

This specification and implementation work is still undergoing in the project.

\section{GEO-NETWORKING STACK IMPLEMENTATION IN NS-3}

For the development of the c2c stack in ns-3, the node structure is analyzed. An overview of ns-3 conceptual implementation architecture can be observed in Fig. 3. As depicted in Fig. 3, any communication stack in ns-3 needs to be attached on similar guidelines. Depending on the simulation scenario and application requirements, the appropriate stack will attach itself to an ns-3 node. Apart from the c2c stack structure in ns-3, iTETRIS would incorporate in ns-3 a geographical 


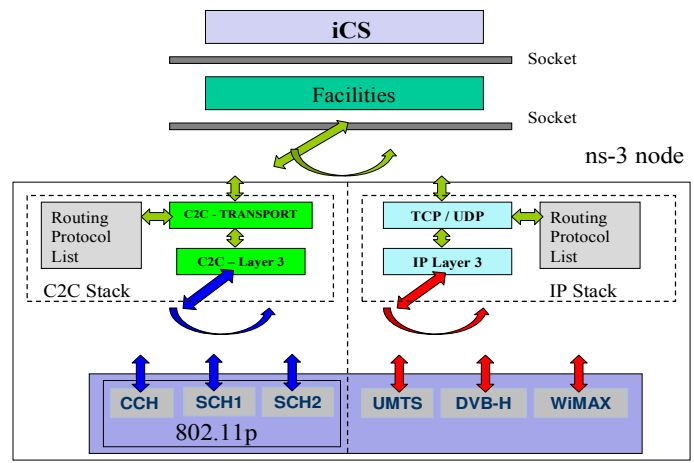

Fig. 3: C2C stack implementation in ns-3: conceptual overview

based addressing scheme, relevant geonetworking headers/packets' structure and modification of the tracing mechanisms. Depending to the application requirements and network congestion status, a specific communication stack and/or access technologies can be selected, and accordingly activated by facilities. This communication channel management is one of the most important functions being defined in ETSI TC ITS. This platform benefits from the modularity and extensibility in ns-3 and aims to serve as a 'de-facto' platform for ITS research on network simulation. Last but not the least, a socket based interface needs to be implemented in order to couple ns-3 and iCS.

iTETRIS Adaptation of a general ns-3 node structure is illustrated as in Fig. 5. The implemented structure can be explained by considering the flow of information from iCS to the 'Message Management' entity in an ns-3 node, which is the first component which receives information/commands from iCS based on application requirements. The 'Message Management' passes the information to the 'StackHandler' which queries 'Communication Channel Selector' and receives back the communication channel identifier which symbolizes assignment of a specific path (Combination of Transport Protocol, Routing Protocol, Network Protocol, Access Technology) to the message.

In order to maintain the extendibility of ns-3 platform for further medication when standards are finalized and incorporation of other advanced protocols, iTETRIS partners decides to externalize the networking and/or transportation protocols and other advanced functionalities from the basic implementation ns-3 architecture.

The basic architecture implementation is recently achieved within the project. Advanced protocols and functionalities are planned in the next phase of implementation.

\section{CONCLUSION}

In context of iTETRIS, the paper highlights the constraints and issues associated with large-scale cooperative systems simulations. It also demonstrates mechanisms to adapt conventional protocols and existing simulators in synchronization with ongoing standardization activities for achieving close to real simulations with such capabilities and magnitude.

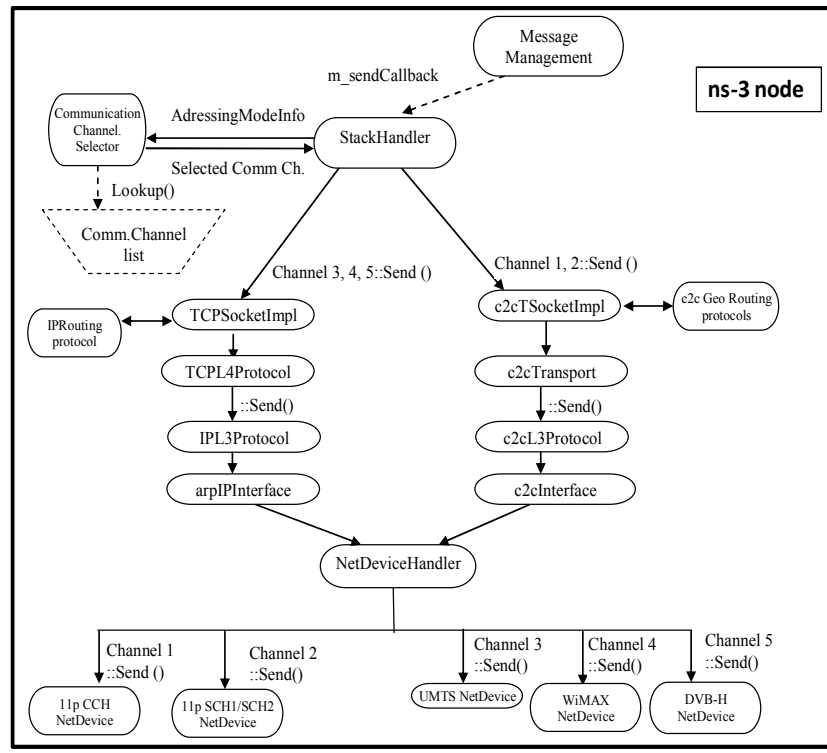

Fig. 5: C2C stack implementation in ns-3: Implementation flow

\section{REFERENCES}

[1] http://www.ict-itetris.eu/iTETRIS project official website.

[2] ETSI TC ITS

[3] http://sumo.sourceforge.net/; last accessed on 17.02.2010

[4] Daniel Krajzewicz, Michael Bonert, and Peter Wagner. The Open Source Traffic Simulation Package SUMO. In RoboCup 2006 Infrastructure Simulation Competition, Bremen, Germany, 2006

[5] Network Simulator, ns-3: http://www.nsnam.org/; last accessed on 17.02.2010

[6] T. R. Henderson, M. Lacage, and G. F. Riley.Network Simulations with the ns-3 Simulator. Demo paper at ACM SIGCOMM'08, August 2008.

[7] Piórkowski M, Raya M et al (2008) TraNS: Realistic Joint Traffic and Network Simulator for VANETs. SIGMOBILE Mob. Comput. Commun. Rev., Vol 12, No 1, Year 2008. ISSN 1559-1662. Pages 31-33. doi: http://doi.acm.org/10.1145/1374512.1374522

[8] EPFL. Trans wb site. http://trans.epfl.ch; last accessed on 16.02.2010

[9] INFRAS, "Handbook Emission Factors for Road Transport", http://www.hbefa.net/; last accessed on 17.02.2010

[10] IMAGINE Consortium, IMAGINE web page, http://www.imagine-project.org/

[11] Wegener A, Piórkowski M, et al (2008) TraCI: An Interface for Coupling Road Traffic and Network Simulators. In: Proceedings of the 11th Communications and Networking Simulation Symposium (CNS'08), Ottawa, Canada

[12] Wegener A, Hellbrück M, et al (2008) VANET Simulation Environment with Feedback Loop and its Application to Traffic Light Assistance. In: Proceedings of the 3rd IEEE Workshop on Automotive Networking and Applications, New Orleans, LA, USA

[13] http://www.isi.edu/nsnam/ns/ns-2 network simulator official website

[14] http://www.omnetpp.org/ OMNeT++ official website

[15] J.D. Day, H Zimmermann. The OSI Reference Model.

[16] http://www.geonet-project.eu GeoNet project official website

[17] Hrizi, Fatma;Filali, Fethi. Achieving broadcasting efficiency in V2X networks with a simple distance-based protocol.: COMNET 2009, 1st International Conference on Communications and Networking, November, 2009, Hammamet, Tunisia. 